\title{
Article \\ Accuracy of Master Casts Generated Using Conventional and Digital Impression Modalities: Part 2-The Full Arch Dimension
}

\author{
Mohammed E. Sayed ${ }^{1,2, * \mathbb{C}}$, Hosain Al-Mansour ${ }^{3}$, Abdulkarim Hussain Alshehri ${ }^{1}$, Fuad Al-Sanabani ${ }^{1}$, \\ Bandar M. A. Al-Makramani ${ }^{1}$ (), Maryam Hassan Mugri ${ }^{4}$, Walaa Magdy Ahmed ${ }^{5}{ }^{(0}$, Nasser M. Alqahtani ${ }^{6}$, \\ Dalea Mohammed Bukhary ${ }^{7}{ }^{(1)}$, Fatimah H. Alsurayyie ${ }^{1}$, Fawzia Ibraheem Shaabi ${ }^{1}$, Yasir Yahya Akkam ${ }^{8}$, \\ Hanan Ibrahim Hakami ${ }^{9}$ and Saurabh Jain $1, *$ (1)
}

Citation: Sayed, M.E.; Al-Mansour, H.; Alshehri, A.H.; Al-Sanabani, F.;

Al-Makramani, B.M.A.; Mugri, M.H.; Ahmed, W.M.; Alqahtani, N.M.; Bukhary, D.M.; Alsurayyie, F.H.; et al. Accuracy of Master Casts Generated Using Conventional and Digital Impression Modalities: Part 2-The Full Arch Dimension. Appl. Sci. 2022, 12, 2148. https://doi.org/10.3390/ app12042148

Academic Editor: Joseph Nissan

Received: 3 January 2022

Accepted: 15 February 2022

Published: 18 February 2022

Publisher's Note: MDPI stays neutral with regard to jurisdictional claims in published maps and institutional affiliations.

Copyright: (C) 2022 by the authors. Licensee MDPI, Basel, Switzerland. This article is an open access article distributed under the terms and conditions of the Creative Commons Attribution (CC BY) license (https:// creativecommons.org/licenses/by/ $4.0 /)$.
1 Department of Prosthetic Dental Sciences, College of Dentistry, Jazan University, Jazan 45142, Saudi Arabia; ahalshehri@jazanu.edu.sa (A.H.A.); fualsanabani@jazanu.edu.sa (F.A.-S.);

bal-makramani@jazanu.edu.sa (B.M.A.A.-M.); falsoraiee@jazanu.edu.sa (F.H.A.); fshaabi@jazanu.edu.sa (F.I.S.)

2 Rutgers School of Dental Medicine, Rutgers University, Newark, NJ 07103, USA

3 Department of Dental Implant, Riyadh Dental Center, King Saud Medical City, Riyadh 12746, Saudi Arabia; Hosain.almansour@student.riyadh.edu.sa

4 Department of Maxillofacial Surgery and Diagnostic Sciences, College of Dentistry, Jazan University, Jazan 45142, Saudi Arabia; mmugri@jazanu.edu.sa

5 Department of Restorative and Aesthetic Dentistry, Faculty of Dentistry, King Abdulaziz University, Jeddah 21589, Saudi Arabia; wmahmed@kau.edu.sa

6 Department of Prosthetic Dental Science, College of Dentistry, King Khalid University, Abha 61413, Saudi Arabia; nmalqahtani@kku.edu.sa

7 Oral and Maxillofacial Prosthodontics Department, Faculty of Dentistry, King Abdulaziz University, Jeddah 21589, Saudi Arabia; dbukhary@kau.edu.sa

8 Isawiyah General Hospital, Qurayat 77413, Saudi Arabia; akkamyasir1414@gmail.com

9 Ministry of Health, Jazan 45142, Saudi Arabia; ihananhakami991@gmail.com

* Correspondence: drsayed203@gmail.com (M.E.S.); drsaurabhjain79@gmail.com (S.J.)

Abstract: The aim of this study was to evaluate the accuracy of master casts generated by conventional (putty and light body consistencies polyvinyl siloxane and alginate) and digital impression techniques on a typodont master model with full-arch-prepared abutment teeth. The null hypotheses tested were as follows: (1) no statistically significant differences in accuracy between casts made by the two impression modalities and the typodont master model at each of the four locations (horizontal straight, horizontal curved, horizontal cross arch, and vertical), and (2) no statistically significant differences in dimensions measured at each of the four locations between the casts generated using the conventional and digital impression techniques. For the conventional technique, 10 impressions each were made for the typodont model using polyvinyl siloxane and alginate impression materials, and the casts were poured. For the digital technique, the typodont model was scanned 10 times using a TRIOS-3 3Shape intraoral scanner, and the casts were printed. The measurements for the horizontal (anteroposterior and cross arch) and vertical dimensions were made using a stereomicroscope and the accuracy of fabricated casts was expressed as the percentage of deviation from the typodont master model's values. A one-way ANOVA and Tukey's test $(p<0.05)$ were used to analyze the data. In the current study, the only measurement that did not exceed $0.5 \%$ in dimensional change was with the stone casts produced by both the 3M ESPE PVS and Kromopan alginate impression materials at the HAPC dimension. The casts generated by impressions made using the 3M ESPE PVS impression material were the most accurate, whereas the casts generated by making digital impressions using the TRIOS-3 3Shape intraoral scanner were the least accurate among the three tested groups. The greatest number of distortions above $0.5 \%$ (at all dimensional locations) was produced by the digital models printed using the ASIGA 3D printer.

Keywords: accuracy; alginate; impression; intraoral scanner; polyvinyl siloxane; digital impression; intraoral scanner 


\section{Introduction}

Producing digital impressions with dental scanners has become common practice in the field of fixed prosthodontics. The digital impression does not employ impression materials or need to be poured, disinfected, and shipped to the dental laboratory. As a consequence, this eliminates the associated problems, leading to a possible improvement in the quality of impressions [1,2]. It has been concluded that digital impression techniques are clinically acceptable for the fabrication of crowns and short-span FPD, but the conventional impression technique is still recommended for full arch FPD [3].

A previous in vitro study evaluated the accuracy of four conventional impression materials with four digital impression systems, and reported greater local deviations with digital impression systems [4]. An in vivo study by Flügge et al. attempted to assess the realistic application and precision of digital scanners. The lowest precision was reported for iTero full-arch intraoral scans, followed by extra-oral model scanning with iTero scans whereas stone model scanning with extra-oral scanner D250 3Shape showed the highest precision [5].

Studies conducted by Ender and Mehl [6], and Mühlemann et al. [7] reported a higher precision of conventional impressions when compared to the digital ones, whereas comparable precision was also reported for quadrant impressions made using digital and conventional techniques, but a significant difference in precision was reported between various digital scanners used in the study [8]. Moreover, a previous in vitro study reported comparable accuracy of full-arch digital impressions when compared to conventional impressions [9].

In their in vivo study on full arch impressions, Ender et al. reported higher precision with conventional impressions materials compared to the eight digital intraoral scanners used in their study. They reported that full dental arches could be successfully recorded by all eight intraoral scanners [10]. A similar clinical study by Gan et al. also supported the use of intraoral digital scanners for generating maxillary full-arch impressions with acceptable accuracy [11].

The amount of distortion in the stone cast produced by conventional impression differs according to the clinical case and the location of the prepared abutment teeth (i.e., short span in one quadrant, long span crossing the midline, or in some cases full mouth). Despite the different concepts between the conventional and digital impression methods, they are used for reproducing oral hard and soft tissues. This current study was conducted to evaluate the accuracy of master cast generated by conventional (putty and light body consistencies polyvinyl siloxane (PVS) and alginate (ALG)) and digital impression techniques on a typodont master model with full-arch-prepared abutment teeth. This study examined the following two null hypotheses: (1) no statistically significant differences in accuracy between casts made by the two impression modalities and the typodont master model at each of the four locations (horizontal straight (HS), horizontal curved (HC), horizontal cross arch (HCA), and vertical (V)), and (2) no statistically significant differences in dimensions measured at each of the four locations between the casts generated using the conventional and digital impression techniques.

\section{Materials and Methods}

\subsection{Materials}

The materials included in the present study are listed in Table 1. 
Table 1. Commercial names and details of the materials used in the study.

\begin{tabular}{ccc}
\hline Material & Manufacturer & Notes \\
\hline $\begin{array}{c}\text { Putty consistency PVS } \\
\text { impression material }\end{array}$ & $\begin{array}{c}\text { Express STD, 3M ESPE, Seefeld, } \\
\text { Germany, batch no. NA77786 }\end{array}$ & $\begin{array}{c}\text { Used with the } \\
\text { recommended tray adhesive }\end{array}$ \\
\hline $\begin{array}{c}\text { Light body } \\
\text { consistency PVS } \\
\text { impression material }\end{array}$ & $\begin{array}{c}\text { Express, 3M ESPE, Seefeld, } \\
\text { Germany, batch no. N653902 }\end{array}$ & $\begin{array}{c}\text { Used with the } \\
\text { recommended tray adhesive }\end{array}$ \\
\hline Alginate & Kromopan, Lascod, Florence, Italy \\
3D print dental model resin & ASIGA Dental MODEL, lot no. \\
& MO/10259, Asiga Pty Ltd., \\
& Alexandria, NSW, Australia & \\
\hline & Elite Rock, Badia Polesine, \\
Type IV die stone & Zhermack Rovigo, Italy, \\
& no. 0000310633 & \\
\hline
\end{tabular}

\subsection{Preparation of the Typodont Master Model}

A high-speed handpiece was fixed to a customized dental surveyor to perform standardized all-ceramic preparations in the typodont models [12]. The preparation was smoothed, and all sharp points or line angles were removed. Guidelines for the preparation are listed in Table 2.

For recording the horizontal measurements, reference points in the form of parallel indentations were milled on the prepared teeth $(1.7,1.5,2.1$, and 2.7) on the typodont master model. The horizontal measurements that were recorded were as follows: horizontal straight $(1.7,1.5)$, horizontal curved $(17,2.1)$, and horizontal cross-arch $(1.7,2.7 ;$ Figure 1A). For recording the vertical measurements, parallel occlusal and cervical reference points were milled on prepared teeth 2.3 (Figure 1B).

Table 2. Guidelines for preparation of typodont teeth.

\begin{tabular}{cc}
\hline \multicolumn{1}{c}{ Anterior Teeth } \\
\hline Typodont teeth prepared \\
Incisal reduction \\
Axial reduction (facial, palatal, mesial, and distal) \\
Circumferential shoulder width \\
Missing teeth & $1.3,1.1,2.1,2.3$ \\
\multicolumn{1}{c}{ Posterior Teeth } & $2 \mathrm{~mm}$ \\
& $1.2-1.4 \mathrm{~mm}$ \\
Typodont teeth prepared & $1 \mathrm{~mm}$ \\
Occlusal reduction & $1.2,2.2$ \\
\hline Axial reduction (buccal, palatal, mesial, and distal) \\
Circumferential shoulder width \\
Missing teeth
\end{tabular}



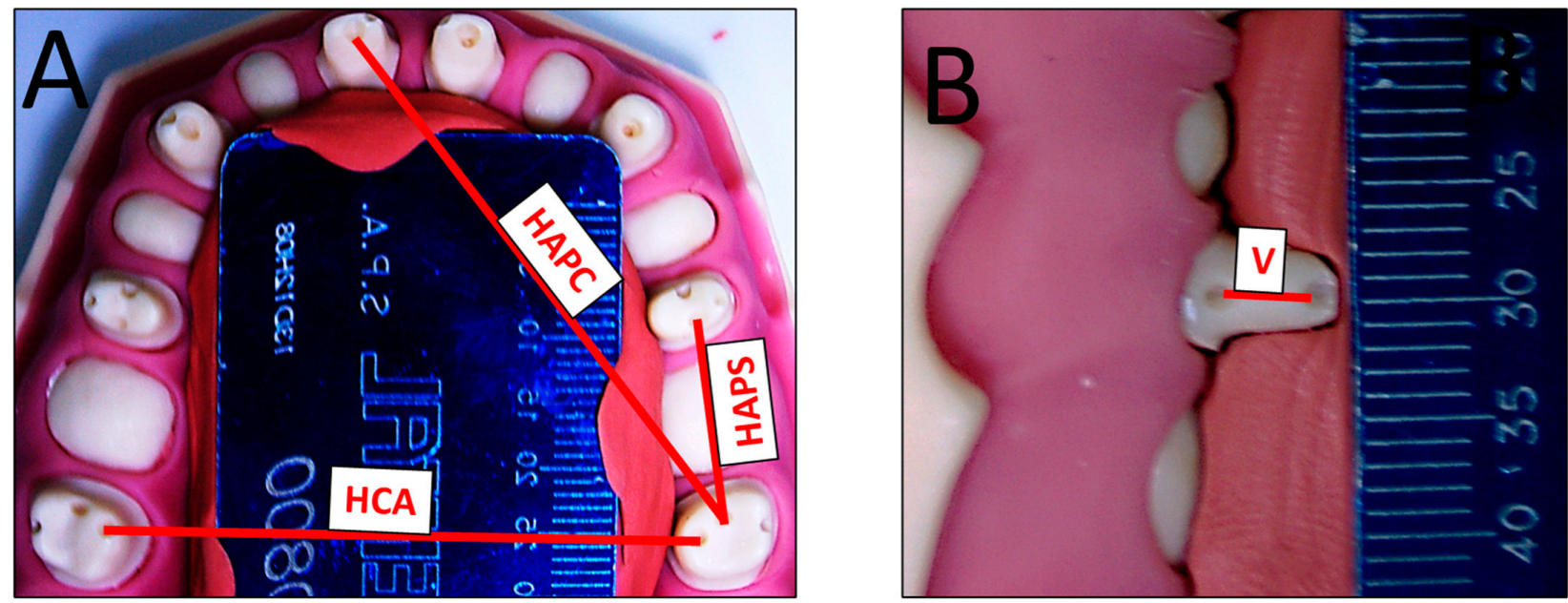

Figure 1. Master model with prepared teeth and index points for (A) horizontal and (B) vertical measurements, in which the positioning of the ruler was performed for digital calibration. HAPShorizontal anteroposterior straight; HAPC — horizontal anteroposterior curved; HCA—horizontal cross-arch; V-vertical. Note: images taken by the stereomicroscope are mirrored.

\subsection{Fabrication of Casts Using the Conventional and Digital Impression Techniques}

The steps involved in the fabrication of casts using conventional and digital impression techniques are explained in Figures $2-5$.

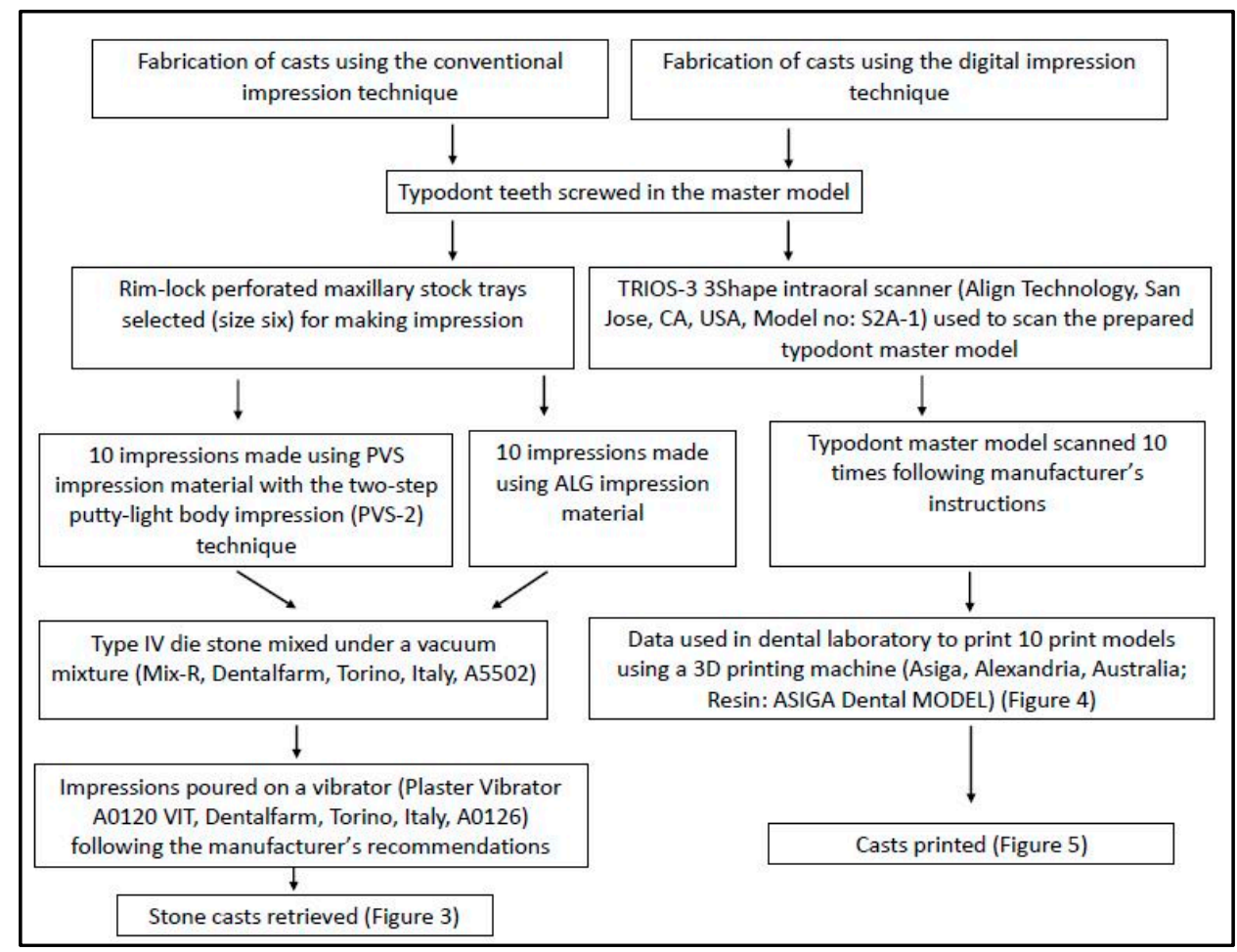

Figure 2. Steps involved in the fabrication of casts using the conventional and digital impression techniques. 


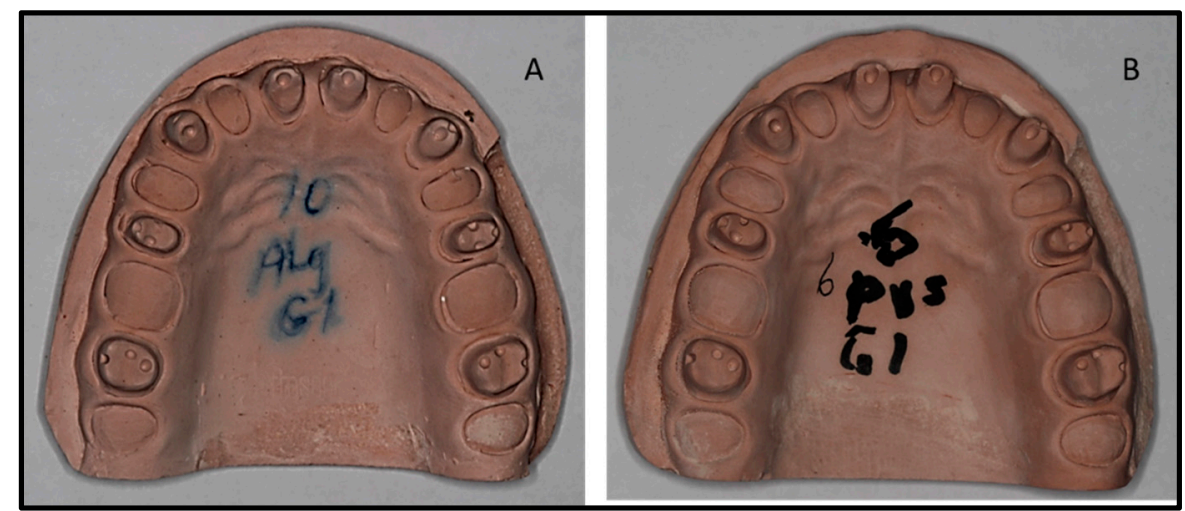

Figure 3. Casts fabricated using the conventional impression techniques: (A) alginate impression and (B) PVS impression.

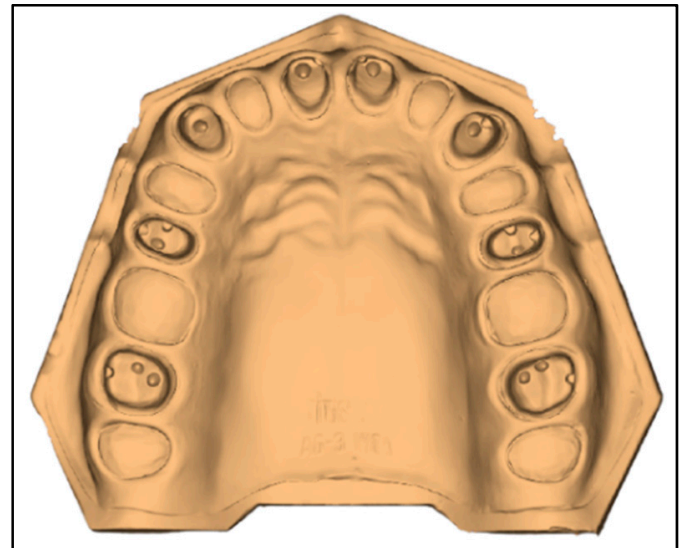

(A)

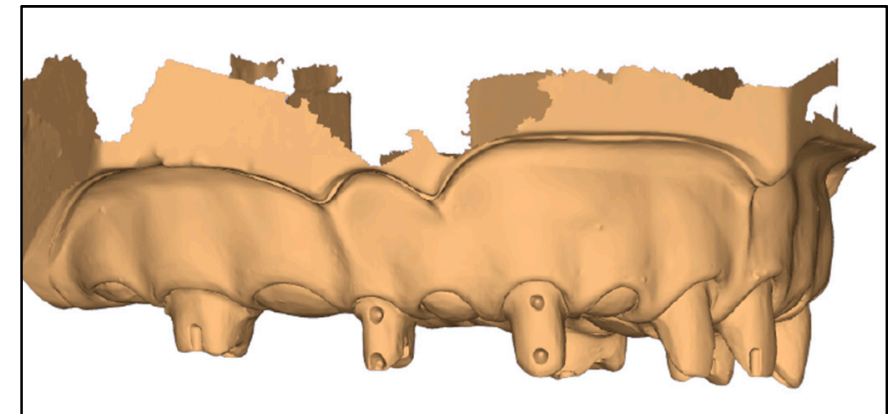

(B)

Figure 4. (A,B) Scanned images of the prepared typodont master model.

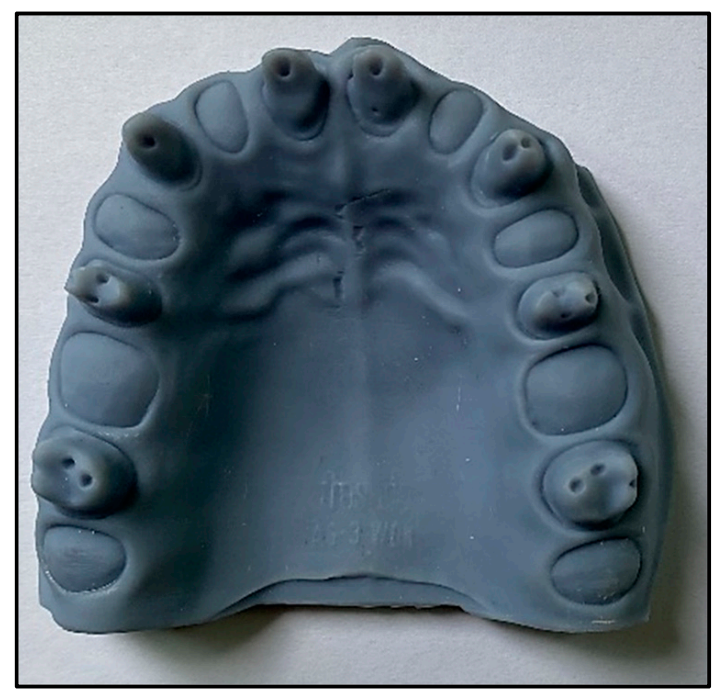

Figure 5. Cast fabricated using the digital impression technique and 3D printing.

\subsection{Measurements}

The measurements for the horizontal (anteroposterior and cross arch) and vertical dimensions were made using a stereomicroscope with a connected USB CCD camera (Amscope, Irvine, CA, USA), personal computer, and compatible measurement software 
(Version no. 3.7.12924). Putty indexes were used to ensure the same distance and angulation from the camera. Typodont master models were measured three times for the horizontal and vertical distances and the average obtained was used as a control while comparing with two groups of poured stone and printed master casts. Each cast was measured three times and the average values were tabulated. The accuracy of casts fabricated by both conventional and digital methods was expressed as the percentage of deviation from the typodont master model's values.

\subsection{Data Analysis}

Descriptive statistics were used to calculate the mean and standard deviations. Oneway analysis of variance (ANOVA) was used to assess the significance of the differences in the dimensional measurements of the casts generated by conventional and digital impression techniques. For pairwise comparisons, Tukey's HSD test was used to determine the differences between the tested subgroups. Moreover, a one-sample $t$-test was used to analyze the dispersion of the measurements around the fixed values of the typodont master model. Data were processed using SPSS software for Windows, version 21 (SPSS Inc., Chicago, IL, USA). For all the statistical analyses, the level of significance was set at $p<0.05$. In addition, according to the American Dental Association (ADA) specification no. 19 , the accuracy of the fabricated casts is expressed as the percentage of deviation from the corresponding typodont master model's values. For each dimension, the difference between the mean value of the cast model (MCM) and the mean value of the typodont master model (MTMM), divided by the mean value of the typodont master model and multiplied by 100, expressed as the percentage of deviation from the typodont master model for each test subgroup of each dimension, was computed as follows:

$$
\text { Percentage of deviation }=[(\mathrm{MCM}-\mathrm{MTMM}) / \mathrm{MTMM})] \times 100
$$

\section{Results}

Table 3 provides the descriptive statistics reported on the stone casts and digitally printed models from the three tested groups (ALG, PVS-2, and DIGITAL) and typodont master cast in each dimension (HAPS, HAPC, HCA, and V).

Table 3. Measurements of stone casts and typodont master casts (mm).

\begin{tabular}{ccccccccc}
\hline & \multicolumn{2}{c}{ Typodont Model } & \multicolumn{2}{c}{ ALG } & \multicolumn{2}{c}{ PVS-2 } & \multicolumn{2}{c}{ DIGITAL } \\
\hline Dimension (mm) & Mean & SD & Mean & SD & Mean & SD & Mean & SD \\
\hline HAPS & 15.100 & 0.00 & $15.313^{*}$ & 0.03 & $15.188^{*}$ & 0.04 & $15.567^{*}$ & 0.05 \\
HAPC & 41.538 & 0.00 & $41.406^{*}$ & 0.08 & $41.387^{*}$ & 0.05 & $41.748^{*}$ & 0.05 \\
HCA & 45.019 & 0.00 & $45.322^{*}$ & 0.06 & $45.264^{*}$ & 0.06 & $45.279 *$ & 0.05 \\
Vertical & 4.310 & 0.00 & $4.082^{*}$ & 0.11 & $4.113^{*}$ & 0.01 & $4.096^{*}$ & 0.01 \\
\hline
\end{tabular}

* Significant compared to typodont master model at $p \leq 0.05$. SD-standard deviation; ALG-alginate; PVS-2— two-step putty-wash polyvinyl siloxane; HAPS - horizontal anteroposterior straight; HAPC—horizontal anteroposterior curved; HCA-horizontal cross-arch.

Testing of the first null hypothesis required three comparisons (ALG vs. typodont master cast, PVS-2 vs. typodont master cast, and digitally printed models vs. typodont master cast) for each dimension (Table 4). Pairwise comparisons were carried out using $t$-tests and revealed that the horizontal anteroposterior straight (HAPS), horizontal anteroposterior curved (HAPC), horizontal cross-arch (HCA), and vertical (V) dimensions on the typodont master cast and stone casts generated from the ALG and PVS-2 impression materials and digital models were significantly different $(p<0.001)$. In general, all measurements on stone casts produced by ALG and PVS-2 impression materials were significantly lower than those for the master cast $(p<0.001)$ at HAPC and V dimensions, but were significantly higher than those for the master cast $(p<0.001)$ at HAPS and HCA dimensions, whereas the measurements on the digitally printed models were significantly higher than those for 
the master cast $(p<0.001)$ at HAPS, HAPC, and HCA dimensions, but were significantly lower than those for the master cast $(p<0.001)$ at the $\mathrm{V}$ dimension.

Table 4. One sample $t$-test for the dispersion of measurements around the test values (typodont master cast measurements).

\begin{tabular}{cccc}
\hline \multicolumn{5}{c}{ Horizontal Anteroposterior Straight Test Value = 15.10 } \\
\hline t (df) & Mean Diff. (95\% CI) & $p$-Value \\
\hline ALG & $24.39(9)$ & $0.21(0.19,0.23)$ & $<0.001$ \\
PVS-2 & $28.93(9)$ & $0.09(0.06,0.11)$ & $<0.001$ \\
DIGITAL & $18.61(9)$ & $0.47(0.43,0.50)$ & $<0.001$ \\
\hline ALG & Horizontal Anteroposterior Curved Test Value $=41.54$ \\
PVS-2 & $-5.31(9)$ & $-0.13(-0.19,-0.08)$ & $<0.001$ \\
DIGITAL & $-9.43(9)$ & $-0.15(-0.19,0.12)$ & $<0.001$ \\
ALG & $12.94(9)$ & $0.21(0.17,0.25)$ & $<0.001$ \\
PVS-2 & Cross Arch Test Value $=45.02$ & $<0.001$ \\
DIGITAL & $14.85(9)$ & $0.30(0.26,0.35)$ & $<0.001$ \\
& $12.13(9)$ & $0.24(0.20,0.29)$ & $<0.001$ \\
\hline ALG & $18.19(9)$ & $0.26(0.23,0.29)$ & $<0.001$ \\
PVS-2 & Vertical Test Value $=4.31$ & $<0.001$ \\
DIGITAL & $-6.72(9)$ & $-0.23(-0.31,-0.15)$ & $<0.001$ \\
\hline
\end{tabular}

ALG—alginate; PVS-2 —-two-step putty-wash polyvinyl siloxane; $\mathrm{df}$-degrees of freedom.

To test the second hypothesis, one-way ANOVA was conducted to examine the differences in accuracy of the casts made by the two impression materials and the digital system. According to Table 5, there was a significant difference in means only at the HAPS and HAPC dimensions location $(p<0.001)$, but there was no significant difference in means at the HCA and V dimensions location $(p>0.05)$.

Table 5. Comparison of dimensional accuracy $(\mathrm{mm})$ between ALG, PVS-2, and digitally printed models using one-way ANOVA.

\begin{tabular}{|c|c|c|c|c|c|c|}
\hline Dimension & Cement & $n$ & Mean & SD & $\begin{array}{c}\text { F Statistics } \\
\text { (df) }\end{array}$ & $p$-Value ${ }^{a}$ \\
\hline \multirow{3}{*}{ HAPS } & ALG & 10 & 15.31 & 0.03 & \multirow{3}{*}{$\begin{array}{c}236.39 \\
(2.27)\end{array}$} & \multirow{3}{*}{$<0.001$} \\
\hline & PVS-2 & 10 & 15.19 & 0.04 & & \\
\hline & DIGITAL & 10 & 15.57 & 0.05 & & \\
\hline \multirow{3}{*}{ HAPC } & ALG & 10 & 41.41 & 0.08 & \multirow{3}{*}{$\begin{array}{c}108.52 \\
(2.27)\end{array}$} & \multirow{3}{*}{$<0.001$} \\
\hline & PVS-2 & 10 & 41.39 & 0.05 & & \\
\hline & DIGITAL & 10 & 41.75 & 0.05 & & \\
\hline \multirow{3}{*}{ HCA } & ALG & 10 & 45.32 & 0.06 & \multirow{3}{*}{$\begin{array}{c}2.67 \\
(2.27)\end{array}$} & \multirow{3}{*}{0.088} \\
\hline & PVS-2 & 10 & 45.26 & 0.06 & & \\
\hline & DIGITAL & 10 & 45.28 & 0.04 & & \\
\hline \multirow{3}{*}{$\mathrm{V}$} & ALG & 10 & 4.08 & 0.04 & \multirow{3}{*}{$\begin{array}{c}0.62 \\
(2.27)\end{array}$} & \multirow{3}{*}{0.543} \\
\hline & PVS-2 & 10 & 4.11 & 0.05 & & \\
\hline & DIGITAL & 10 & 4.10 & 0.05 & & \\
\hline
\end{tabular}

a One-way ANOVA was used. Significant level was set at 0.05. The significance level was set at 0.05. SD—standard deviation; ALG—alginate; PVS-2—two-step putty-wash polyvinyl siloxane; HAPS—horizontal anteroposterior straight; HAPC — horizontal anteroposterior curved; HCA-horizontal cross-arch; V—vertical; df—-degrees of freedom; ANOVA—analysis of variance.

Further analysis using Tukey's HSD post hoc test was conducted among the three groups in the two dimensions location (HAPS and HAPC; Table 6). A statistically significant difference in the two dimensions (HAPS and HAPC) of the stone casts was detected among the three groups (ALG, PWAS-2, and CAD/CAM) (exception between ALG and PVS-2 at the HAPC dimension). In general, casts made from both ALG and PWAS-2 impression 
materials experienced contraction at the HAPC and V dimensions but underwent expansion at the HAPS and HCA dimensions. However, digitally printed models showed expansion at HAPS, HAPC, and HCA, but underwent contraction at the V dimension.

Table 6. Multiple pairwise comparisons of the accuracy of the two impression materials and digitally printed models using Tukey's HSD test.

\begin{tabular}{|c|c|c|c|c|}
\hline & Pairwise Comparison & Mean (SD) & Mean Difference & $p$-Value ${ }^{\text {a }}$ \\
\hline \multirow{3}{*}{ HAPS } & ALG vs. PVS-2 & $\begin{array}{l}15.31(0.03) \\
15.19(0.04)\end{array}$ & 0.12 & $<0.001 *$ \\
\hline & ALG vs. DIGITAL & $\begin{array}{l}15.31(0.03) \\
15.57(0.05)\end{array}$ & -0.26 & $<0.001$ * \\
\hline & PVS-2 vs. DIGITAL & $\begin{array}{l}15.19(0.04) \\
15.57(0.05)\end{array}$ & -0.38 & $<0.001$ * \\
\hline \multirow{3}{*}{ HAPC } & ALG vs. PVS-2 & $\begin{array}{l}41.41(0.08) \\
41.39(0.05)\end{array}$ & 0.02 & 0.772 \\
\hline & ALG vs. DIGITAL & $\begin{array}{l}41.41(0.08) \\
41.75(0.05)\end{array}$ & -0.34 & $<0.001$ * \\
\hline & PVS-2 vs. DIGITAL & $\begin{array}{l}41.39(0.05) \\
41.75(0.05)\end{array}$ & -0.36 & $<0.001$ * \\
\hline
\end{tabular}

a A one-way ANOVA was used, * The mean difference is significant at the 0.05 level. SD-standard deviation; ALG—alginate; HAPS—horizontal anteroposterior straight; HAPC—horizontal anteroposterior curved; HCAhorizontal cross-arch; V-vertical.

Table 7 shows the percentage of deviations (\%) and absolute changes $(\mu \mathrm{m})$ of dimensions of stone casts from those of the typodont master cast for the three tested groups.

Table 7. The percentage of deviation (\%) and absolute change $(\mu \mathrm{m})$ from the typodont master cast.

\begin{tabular}{cccccccccc}
\hline & \multicolumn{2}{c}{ HAPS } & \multicolumn{2}{c}{ HAPC } & \multicolumn{2}{c}{ HCA } & \multicolumn{2}{c}{$\mathrm{V}$} \\
\hline $\begin{array}{c}\text { Type of } \\
\text { Impression }\end{array}$ & $\%$ & $\mu \mathrm{m}$ & $\%$ & $\boldsymbol{\mu m}$ & $\%$ & $\boldsymbol{~} \mathbf{m}$ & $\%$ & $\mu \mathbf{m}$ \\
\hline ALG & 1.41 & 213 & -0.32 & 132 & 0.67 & 303 & -5.29 & 228 \\
PVS-2 & 0.58 & 88 & -0.37 & 151 & 0.54 & 245 & -4.56 & 197 \\
DIGITAL & 3.09 & 467 & 0.50 & 210 & 0.58 & 260 & -4.96 & 214
\end{tabular}

ALG—alginate; PVS-2—two-step putty-wash polyvinyl siloxane; HAPS—horizontal anteroposterior straight; HAPC—-horizontal anteroposterior curved; HCA-horizontal cross-arch; V—vertical.

A dimensional change of more than $0.5 \%$ is reflected as a clinically significant distortion, as per the American Dental Association specification no. 19 for elastomeric type I impressions [13].

Figure 6 summarizes the data for the three groups. The figure demonstrates that the only measurement that did not exceed $0.5 \%$ in dimensional change was the HAPC measurement for stone casts made by both ALG and PVS-2 impression materials and for the digitally printed models. However, the greatest number of distortions above $0.5 \%$ (at HAPS, HCA, and V) was produced by the digitally printed models. 


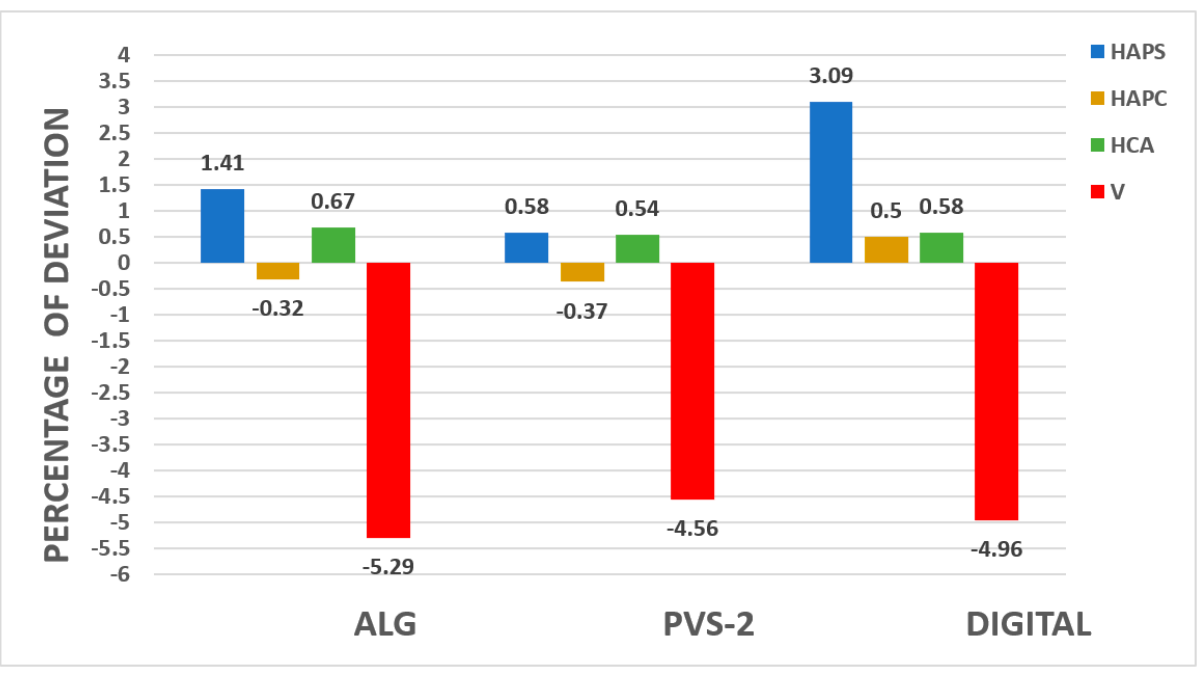

Figure 6. Percentage of deviation from the typodont master cast for the impression materials and CAD/CAM.

\section{Discussion}

In the field of fixed prosthodontics, the rehabilitation of cases with complex cross-archfixed dental prostheses is a difficult procedure. Generating an accurate final impression including the prepared teeth and the entire arch is critical to ensure precise fitting and long-lasting definitive restorations [4]. According to the ADA specification on impression materials, there are specifications to test their accuracy and ability to reproduce fine details using simulated tooth models presenting areas that can be easily measured [14]. The testing conditions embodied in the specifications differ from those encountered during clinical practice; therefore, in the present study, a typodont master model with full-arch-prepared abutment teeth was used.

A TRIOS-3 3Shape intra-oral scanner was used in the current study, as a previous study reported the highest precision for this scanner compared to other intra-oral scanners used in dentistry [13]. As reported in a previous study [12], instead of using digital versions, casts were printed to standardize the groups in order to have the same testing conditions and to avoid any errors, while measurements were made using a stereomicroscope. The distance between the casts and microscope was standardized and a ruler was used for calibration during measurement using the software.

The readings obtained after evaluating the discrepancies of the casts contained both negative and positive values. These were converted to absolute values to avoid false results that could occur due to the positive and negative values canceling each other out $[15,16]$.

Furthermore, in the present study, the die stone casts obtained from PVS-2 presented the highest accuracy compared to the casts obtained from the alginate impression material and digital impressions. This result was supported by other studies that found that the accuracy of full-arch conventional impressions was better than the digital impressions $[4,5,7]$. The high accuracy of the additional silicon two-step putty-light body technique can be explained by the uniform space for the light body material to polymerize with minimal shrinkage, and the details were recorded by the light-body material only [17-19].

The 3D-printed models obtained from the intraoral scanning digital impression did not exhibit superior accuracy compared to the stone casts obtained from the conventional impression materials (ALG and PVS-2). This result is supported by several in vitro studies $[4,5,7,12]$. An in vitro study found that the local deviations of full-arch impressions were higher for digital impression systems than for conventional impression methods [4]. Additionally, it should be noted that most previous studies involved dentulous patients or those with a single missing tooth. However, there is a paucity of literature evaluating the precision of intraoral scanners in partially edentulous cases. 
The present study included a full dental arch with multiple edentulous areas and prepared abutment teeth to imitate the clinical scenario of a complex case. Comparing the result of the present study (full arch) with that of our part 1 study (half-arch), the accuracy of the stone casts produced by both 3M ESPE PVS and the Kromopan alginate impression materials and the digitally printed models was better in the half arch than the full arch [12]. The precision of digital impressions was anticipated to be less in cases where multiple teeth are missing due to a deficiency of reference points from the scanning machine and the dentist [20]. In the current study, the only measurement that did not exceed $0.5 \%$ in dimensional change was with the stone casts produced by both 3M ESPE PVS and Kromopan alginate impression materials at the HAPC dimension.

The results of this study indicated that stone casts generated using conventional impression and pouring techniques have a higher linear dimensional accuracy than 3Dprinted casts, which contradicts the findings reported by recent studies [11,21,22]. A recent systematic review evaluated the accuracy of 3D-printed models generated by different printing technologies and reported that the dimensional variation may exceed $500 \mu \mathrm{m}$, especially for printers that use stereolithographic technology [23]. The 3D printer used in the current study is reported to have a mean discrepancy in the range of 210-467 $\mu \mathrm{m}$, which is comparable to a few other commonly used printers [24]. Such significant variability may be acceptable for diagnostic purposes, but not for prosthodontic procedures that require high levels of precision and accuracy. Inaccuracies up to $50.0 \mu \mathrm{m}$ from the master model were considered to be clinically acceptable as they were unlikely to impede the full seating of a prosthesis $[19,25]$.

In the present study, alginate presented an accuracy comparable to that of PVS-2. This finding is supported by other studies $[15,26]$. In contrast, another study found a high deviation pattern of full-arch alginate impressions that limited its use in dentistry [4]. Furthermore, alginate's instability across time and its surface roughness due to water loss are disadvantages, limiting its use only for diagnostic casts [15].

The type of intraoral scanner used and different scanning conditions can lead to varied results [20]. Moreover, the influence of the scanning approach and selected software on the scanning precision should be verified for each IOS model [27].

The present study is an in vitro study, which differs from the in vivo environment in patients' mouths. Factors like the presence of saliva and/or blood, the patient's and dentist's movements, accessibility in the mouth, and soft tissue stability are the common challenges faced by dentists, which can lead to potential inaccuracies. Therefore, future in vivo studies should be conducted to augment the quality of functioning of the digital workflow.

\section{Conclusions}

Generally, the accuracy of the stone casts produced by the 3M ESPE PVS and Kromopan alginate impression materials and the digitally printed models was better in the previous study (half arch) than in the current study (full arch). In the current study, the only measurement that did not exceed $0.5 \%$ in dimensional change was with the stone casts produced by both 3M ESPE PVS and Kromopan alginate impression materials at the HAPC dimension. The results obtained from this study showed that the casts generated by impressions made using the 3M ESPE PVS impression material were most accurate among the three tested groups. Lastly, we found that the casts generated by making digital impressions using the TRIOS-3 3Shape intraoral scanner were the least accurate among the three groups. The greatest number of distortions above $0.5 \%$ (at all dimensional locations) was produced by the digital models printed using the ASIGA 3D printer. 


\begin{abstract}
Author Contributions: Conceptualization, M.E.S., B.M.A.A.-M., S.J. and F.A.-S.; methodology, M.E.S., B.M.A.A.-M., F.A.-S., H.A.-M. and S.J.; software, B.M.A.A.-M., A.H.A. and F.A.-S.; validation, S.J., W.M.A., N.M.A. and F.A.-S.; formal analysis, M.E.S., B.M.A.A.-M., D.M.B., F.H.A. and H.A.-M.; investigation, M.E.S., B.M.A.A.-M., S.J. and F.A.-S.; resources, M.E.S., H.A.-M., A.H.A., F.I.S., F.H.A., W.M.A., M.H.M., Y.Y.A., H.I.H. and S.J.; data curation, B.M.A.A.-M., F.I.S. and F.A.-S.; writingoriginal draft preparation, M.E.S., B.M.A.A.-M., S.J. and F.A.-S.; writing-review and editing, M.E.S., B.M.A.A.-M., F.A.-S., H.A.-M., A.H.A., F.I.S., F.H.A., W.M.A., N.M.A., D.M.B., M.H.M., Y.Y.A., H.I.H. and S.J.; visualization, B.M.A.A.-M., F.A.-S., A.H.A., F.I.S., F.H.A., W.M.A., D.M.B. and S.J.; supervision, M.E.S., D.M.B., M.H.M., Y.Y.A., H.I.H. and H.A.-M.; project administration, M.E.S. All authors have read and agreed to the published version of the manuscript.
\end{abstract}

Funding: This research received no external funding.

Institutional Review Board Statement: Not applicable.

Informed Consent Statement: Not applicable.

Data Availability Statement: The data that support the findings of this study are available from the corresponding author upon reasonable request.

Conflicts of Interest: The authors declare no conflict of interest.

\title{
References
}

1. Christensen, G.J. The challenge to conventional impressions. J. Am. Dent. Assoc. 2008, 139, 347-349. [CrossRef] [PubMed]

2. Christensen, G.J. Will digital impressions eliminate the current problems with conventional impressions? J. Am. Dent. Assoc. 2008, 139, 761-763. [CrossRef] [PubMed]

3. Ahlholm, P. Digital versus Conventional Impressions in Fixed Prosthodontics: A Review. J. Prosthodont. 2018, 27, 35-41. [CrossRef] [PubMed]

4. Ender, A.; Mehl, A. In-vitro evaluation of the accuracy of conventional and digital methods of obtaining full-arch dental impressions. Quintessence Int. 2015, 46, 9-17.

5. $\quad$ Flügge, T.V.; Schlager, S.; Nelson, K.; Nahles, S.; Metzger, M.C. Precision of intraoral digital dental impressions with iTero and extraoral digitization with the iTero and a model scanner. Am. J. Orthod. Dentofac. Orthop. 2013, 144, 471-478. [CrossRef]

6. Ender, A.; Mehl, A. Accuracy of complete-arch dental impressions: A new method of measuring trueness and precision. J. Prosthet. Dent. 2013, 109, 121-128. [CrossRef]

7. Ender, A.; Attin, T.; Mehl, A. In vivo precision of conventional and digital methods of obtaining complete-arch dental impressions. J. Prosthet. Dent. 2016, 115, 313-320. [CrossRef]

8. Ender, A.; Zimmermann, M.; Attin, T.; Mehl, A. In vivo precision of conventional and digital methods for obtaining quadrant dental impressions. Clin. Oral Investig. 2016, 20, 1495-1504. [CrossRef]

9. Gan, N.; Xiong, Y.; Jiao, T. Accuracy of intraoral digital impressions for whole upper jaws, including full dentitions and palatal soft tissues. PLoS ONE 2016, 11, e0158800. [CrossRef]

10. Mühlemann, S.; Greter, E.A.; Park, J.M.; Hämmerle, C.H.F.; Thoma, D.S. Precision of digital implant models compared to conventional implant models for posterior single implant crowns: A within-subject comparison. Clin. Oral Implant. Res. 2018, 29, 931-936. [CrossRef]

11. Ender, A.; Mehl, A. Full arch scans: Conventional versus digital impressions-An in-vitro study. Int. J. Comput. Dent. 2011, 14, 11-12. [PubMed]

12. Sayed, M.E.; Alshehri, A.H.; Al-Makramani, B.M.A.; Al-Sanabani, F.; Shaabi, F.I.; Alsurayyie, F.H.; Ahmed, W.M.; Al-Mansour, H.; Jain, S. Accuracy of Master Casts Generated Using Conventional and Digital Impression Modalities: Part 1-The Half-Arch Dimension. Appl. Sci. 2021, 11, 12034. [CrossRef]

13. Michelinakis, G.; Apostolakis, D.; Tsagarakis, A.; Kourakis, G.; Pavlakis, E. A comparison of accuracy of 3 intraoral scanners: A single-blinded in vitro study. J. Prosthet. Dent. 2020, 124, 581-588. [CrossRef] [PubMed]

14. Federick, D.R.; Caputo, A. Comparing the accuracy of reversible hydrocolloid and elastomeric impression materials. J. Am. Dent. Assoc. 1997, 128, 183-188. [CrossRef]

15. Chen, S.Y.; Liang, W.M.; Chen, F.N. Factors affecting the accuracy of elastomeric impression materials. J. Dent. 2004, 32, 603-609. [CrossRef]

16. Faria, A.C.; Rodrigues, R.C.; Macedo, A.P.; Mattos Mda, G.; Ribeiro, R.F. Accuracy of stone casts obtained by different impression materials. Braz. Oral Res. 2008, 22, 293-298. [CrossRef]

17. Nissan, J.; Gross, M.; Shifman, A.; Assif, D. Effect of wash bulk on the accuracy of polyvinyl siloxane putty-wash impressions. J. Oral Rehabil. 2002, 29, 357-361. [CrossRef]

18. Dhungana, M.; Acharya, L.; Sah, S. Dimensional accuracy of different impression materials and techniques commonly used in prosthodontics. JNDA 2016, 16, 30-36. 
19. Sayed, M.E.; Al-Makramani, B.M.; Al-Sanabani, F.A.; Mohamed, M.S. Effect of Intermixing Brands on the Dimensional Accuracy of Master Cast using Putty-Wash Impression Technique. J. Contemp. Dent. Pract. 2016, 17, 734-739. [CrossRef]

20. Giachetti, L.; Sarti, C.; Cinelli, F.; Russo, D.S. Accuracy of Digital Impressions in Fixed Prosthodontics: A Systematic Review of Clinical Studies. Int. J. Prosthodont. 2020, 33, 192-201. [CrossRef]

21. Ellakany, P.; Al-Harbi, F.; El Tantawi, M.; Mohsen, C. Evaluation of the accuracy of digital and 3D-printed casts compared with conventional stone casts. J. Prosthet. Dent. 2020. [CrossRef] [PubMed]

22. Aly, P.; Mohsen, C. Comparison of the Accuracy of Three-Dimensional Printed Casts, Digital, and Conventional Casts: An In Vitro Study. Eur. J. Dent. 2020, 14, 189-193. [CrossRef] [PubMed]

23. Etemad-Shahidi, Y.; Qallandar, O.B.; Evenden, J.; Alifui-Segbaya, F.; Ahmed, K.E. Accuracy of 3-Dimensionally Printed Full-Arch Dental Models: A Systematic Review. J. Clin. Med. 2020, 9, 3357. [CrossRef] [PubMed]

24. Reis, B.S.; Portella, F.F.; Rivaldo, E.G. Precision and accuracy of four current 3D Printers to achieve models for Fixed Dental Prosthesis. Precisão de quatro impressoras 3D para obtenção de modelos para prótese fixa. Acta Odontol. Latinoam. 2020, 33, 3-5. [CrossRef] [PubMed]

25. Tjan, A.H.; Nemetz, H.; Nguyen, L.T.; Contino, R. Effect of tray space on the accuracy of monophasic polyvinylsiloxane impressions. J. Prosthet. Dent. 1992, 68, 19-28. [CrossRef]

26. Caputi, S.; Varvara, G. Dimensional accuracy of resultant casts made by a monophase, one-step and two-step, and a novel two-step Putty/light-body impression technique: An in vitro study. J. Prosthet. Dent. 2008, 99, 274-281. [CrossRef]

27. Mandelli, F.; Gherlone, E.F. Full-arch intraoral scanning: Comparison of the two different strategies and their accuracy outcomes. J. Osseointegr. 2018, 10, 65-74. 